\title{
BERNSTEINS THEOREM FOR COMPLETELY EXCESSIVE MEASURES
}

\author{
HEDI BEN SAAD AND KLAUS JANßEN
}

\section{§ 0. Introduction}

Bernstein's theorem states that the following properties are equivalent for a function $\psi:] 0, \infty[\rightarrow \mathbb{R}$ (which then is called completely monotone):

i) $(-1)^{n} L_{h}^{n} \psi \geq 0 \quad$ for all $n \geq 0, h \geq 0$,

ii) $(-1)^{n} D^{n} \psi \geq 0 \quad$ for all $n \geq 0$,

iii) $\psi(t)=\int e^{-s t} \sigma(d s)$ for all $t>0$ for some $\sigma$-finite measure $\sigma$ on $[0, \infty[;$

moreover, the measure $\sigma$ in iii) is uniquely determined.

In these formulas $L_{h}$ is the difference operator given by $L_{h} \psi(t)=$ $\psi(t+h)-\psi(t)$ for $t \in] 0, \infty[, D$ denotes the operator of taking the derivative, and $L_{h}^{n}$ and $D^{n}$ are the iterates of $L_{h}$ and $D$, respectively.

Several authors have obtained generalizations of this result to potential theoretic settings under various regularity assumptions (cf. [4], [9], [10] and references in these papers). We give in this paper a proof for a corresponding result for measures $m$ which are completely excessive with respect to a general semigroup $\left(P_{t}\right)_{t>0}$ of kernels on a nice space (cf. Theorem 2.2), extending in this way results which were obtained by Itô in [9] and by Beznea in [4]. In this result the operator $L_{h}$ is replaced by $\left(P_{t+h}-P_{t}\right)_{t>0}, D$ is replaced by the generator of the semigroup (we only consider the integrated version of ii)), and we obtain an integral representation of $m$ as a mixture of eigenmeasures for $\left(P_{t}\right)$ (which replace the exponentials of iii) above). The proof is based on an easy extension of Bernstein's theorem for measure valued functions (cf. Proposition (1.4)), which allows to avoid Choquet's theorem which was central in [9] and [4]. The proofs are very simple; the only non-obvious result we use is the

Received September 21, 1988. 
original above Bernstein's theorem and a well known result on regularization of pseudo-kernels or the fact that bimeasures on nice spaces are in fact measures which admit a disintegration.

The above Bernstein's theorem concerns functions $\psi$ which are completely excessive functions for the semigroup of uniform motion to the right on $] 0, \infty[$. Our result on completely excessive measures transforms into a result on general completely excessive functions if the potential kernel $U=\int P_{t} d t$ is absolutely continuous with respect to some measure (cf. Remark (2.3.b)).

\section{$\S 1$. Bernstein's theorem for measure valued functions}

This result will be a consequence of a transformation of an integral representation into a factorization of kernels.

Let $(S, \mathscr{S})$ and $(T, \mathscr{T})$ be measurable spaces, let $g: S \times T \rightarrow \overline{\mathbb{R}}_{+}$be a $\mathscr{S} \otimes \mathscr{T}$-measurable function (which will be denoted by $g \in(\mathscr{S} \otimes \mathscr{T})_{+}$), and let $\mathscr{H}$ be a set of positive functions on $T$ such that the following is true: For every $h$ in $\mathscr{H}$ there exists a unique $\sigma$-finite measure on $\sigma_{h}$ on $(S, \mathscr{S})$ such that

$$
h(t)=\int g(s, t) \sigma_{h}(d s) \quad \text { for all } t \text { in } T .
$$

Finally, let $(E, \mathscr{E})$ be a $U$-space (i.e. $E$ is a universally measurable subset of a compact metric space, equipped with its borel field $\mathscr{E}$ ), and let $M: \mathscr{E}_{+} \rightarrow \mathscr{T}_{+}$be a kernel such that $M 1_{A} \in \mathscr{H}$ for all $A$ in $\mathscr{E}$.

We denote by $\sigma$ the unique $\sigma$-finite measure on $(S, \mathscr{S})$ such that $M 1(t)=\int g(s, t) \sigma(d s)$ for all $t$ in $T$, and we define the kernel $N: \mathscr{S}_{+} \rightarrow \mathscr{T}_{+}$ by

$$
N \varphi(t):=\int g(s, t) \varphi(s) \sigma(d s) \quad \text { for all } t \text { in } T
$$

for all $\varphi$ in $\mathscr{S}_{+}$.

(1.1) Lemma. Under the above assumptions there exists a stochastic kernel $R: \mathscr{E}_{+} \rightarrow \mathscr{S}_{+}$such that $M=N R$.

If $R^{\prime}$ is another kernel which factorizes $M$ in this way, then there exists a $\sigma$-null set $B \in \mathscr{S}$ such that $R f=R^{\prime} f$ on $S \backslash B$ for all $f$ in $\mathscr{E}_{+}$.

Proof. Denote for $A$ in $\mathscr{E}$ by $\sigma_{A}$ the measure on $(S, \mathscr{S})$ which verifies 


$$
M 1_{A}(t)=\int g(s, t) \sigma_{A}(d s) \quad \text { for all } t \text { in } T
$$

By the uniqueness assumption we have $\sigma_{\phi}=0, \quad \sigma_{E}=\sigma$ and $\sum_{n} \sigma_{A_{n}}=\sigma_{U_{n} A_{n}}$ for every sequence $\left(A_{n}\right)$ in $\mathscr{E}$ of pairwise disjoint sets.

Since $\sigma_{A}+\sigma_{C A}=\sigma$, we can choose by Radon-Nikodym's theorem a density $\varphi_{A} \leq 1$ of $\sigma_{A}$ with respect to $\sigma$ in $\mathscr{S}_{+}$, for every $A$ in $\mathscr{E}$. We choose $\varphi_{E}=1$, and we conclude that for every sequence $\left(A_{n}\right) \subset \mathscr{E}$ of pairwise disjoint sets we have $\sum_{n} \varphi_{A_{n}}=\varphi_{\cup_{n} A_{n}} \sigma$-a.e. Classical results now imply the existence of a kernel $R: \mathscr{E}_{+} \rightarrow \mathscr{S}_{+}$such that $R 1_{A}=\varphi_{A} \sigma$-a.e. (cf. p. 453 in [8]). Obviously, we have $M=N R$.

If $M=N R^{\prime}$ for some other kernel $R^{\prime}$, then $R f=R^{\prime} f \sigma$-a.e. for every $f$ in $\mathscr{E}_{+}$, hence the result concerning uniqueness follows from the fact that $\mathscr{E}$ is countably generated.

(1.2) Remark. Obviously, the measures $\sigma_{A}$ of the above proof determine a bimeasure

$$
(A, B) \longrightarrow \sigma_{A}(B)
$$

on $\mathscr{E} \times \mathscr{S}$, and the above proof shows that such bimeasures extend to a measure on $\mathscr{E} \otimes \mathscr{S}$ which has a disintegration with respect to the projection $(x, s) \rightarrow s$ from $E \times S$ onto $S$. The proof of (1.1) as well as the result on disintegration of bimeasures remain true for more general measurable spaces $(E, \mathscr{E})$ if suitable regularity assumptions are imposed on the relevant measures (cf. [6] and also [2] for a Radon measure setting).

It seems that such disintegrations of bimeasures are helpful in many situations. For example, in [11] this was the decisive step to construct an entrance law for suitable excessive measures.

As a simple application of (1.1) for $T:=] 0, \infty$ [ equipped with it's borel field $\mathscr{T}:=\mathscr{B}(] 0, \infty[)$ we obtain a version of Bernstein's theorem for measure valued functions.

(1.3) Definition. A family $\left(\mu_{t}\right)_{t \in] 0, \infty[}$ of measures on a measure space $(E, \mathscr{E})$ is called a completely monotone family of measures if

i) $\mu_{t}\left(f_{0}\right)<\infty$ for all $t>0$ for some strictly positive $f_{0}$ in $\mathscr{E}_{+}$,

ii) $t \rightarrow \mu_{t}(f)$ is completely monotone for all $f$ in $\mathscr{E}_{+}$below $f_{0}$. 
(1.4) Proposition. If $\left(\mu_{t}\right)_{t>0}$ is a completely monotone family of measures on a $U$-space $(E, \mathscr{E})$, then

$$
\mu_{t}=\int e^{-s t} \rho_{s} \sigma(d s) \quad \text { for all } t>0,
$$

where $\sigma$ is a $\sigma$-finite measure on $\left[0, \infty\left[\right.\right.$ and $\left(\rho_{s}\right)_{s \geq 0}$ is a measurable family of measures on $(E, \mathscr{E})$, i.e. $s \rightarrow \rho_{s}(f)$ is borel on $\mathbb{R}_{+}$for every $f$ in $\mathscr{E}_{+}$.

Moreover, the associated measure $\int \rho_{s} \otimes \varepsilon_{s} \sigma(d s)$ on $E \times[0, \infty[$ is uniquely determined by $\left(\mu_{t}\right)_{t>0}$. If $\sigma$ is fixed, the family $\left(\rho_{s}\right)_{s \geq 0}$ is $\sigma$-a.e. uniquely determined.

Proof. Pick $f_{0}$ in $\mathscr{E}_{+}$strictly positive such that i) and ii) from 1.3 hold. If we denote for $A$ in $\mathscr{E}$ by $M 1_{A}$ the function $t \rightarrow \mu_{t}\left(1_{A} \cdot f_{0}\right)$ on ]0, $\infty$ [, then $M$ extends to a kernel $M: \mathscr{E}_{+} \rightarrow \mathscr{T}_{+}$; by assumption $h:=M 1_{A}$ is a completely monotone function on $] 0, \infty$ [ for $A$ in $\mathscr{E}$, which by the classical Bernstein's theorem can be represented as

$$
h(t)=\int e^{-s t} \sigma_{h}(d s) \quad \text { for } t>0
$$

with a unique $\sigma$-finite measure $\sigma_{h}$ on $(S, \mathscr{S})$ for $S:=[0, \infty$ [ equipped with its borel field $\mathscr{S}$.

Let $\mathscr{H}$ be the set of completely monotone functions on $] 0, \infty[$, and let $\sigma$ be the measure which represents $M 1: t \rightarrow \mu_{t}\left(f_{0}\right)$. Then the assumptions of (1.1) are fulfilled. Let $R$ be the stochastic kernel which factorizes $M$ according to (1.1). The definition $\rho_{s}(A):=R\left(1 / f_{0} 1_{A}\right)(s)$ for $s$ in $[0, \infty[$ and $A$ in $\mathscr{E}$ then gives the result.

(1.5) Remarks. a) In view of Bernstein's theorem the obvious converse of (1.4) is true.

b) Bernstein's theorem has been generalized to much more general semigroups $T$ (cf. [2]) where a decent theory of exponentials has been established. Obviously, the statement and the proof of (1.4) remain valid under such assumptions.

\section{$\S 2$. Completely excessive measures}

In the following let $(E, \mathscr{E})$ be a $U$-space, and let $\left(P_{t}\right)_{t>0}$ be a measurable semigroup of kernels on $(E, \mathscr{E})$, i.e. we have $P_{t} P_{h}=P_{t+h}$ for $t, h>0$ and $(t, x) \rightarrow P_{t} f(x)$ is $\mathscr{B}(] 0, \infty[) \otimes \mathscr{E}$-measurable for $f$ in $\mathscr{E}_{+}$. We make no assumptions on finiteness or vanishing on the kernels $P_{t}$. 
The associated resolvent $\left(U_{\lambda}\right)_{k \geq 0}$ of kernels on $(E, \mathscr{E})$ is given by $U_{2} f(x)=\int_{0}^{\infty} e^{-\lambda t} P_{t} f(x) d t$ for $f$ in $\mathscr{E}_{+}, x$ in $E$, and $\lambda \geq 0$. We set $U:=U_{0}$.

Remember that a $\sigma$-finite measure $m$ on $E$ is called excessive if $m P_{t} \uparrow m$ for $t \downarrow 0$, or equivalently, if $m$ does not charge $\{U 1=0\}$ and satisfies likewise $\left(m P_{t}\right)_{t>0}$ is decreasing or $\left(\lambda m U_{2}\right)_{l>0}$ is increasing (cf. Chap. XII in [7]).

(2.1) Definitions. a) An excessive measure $m$ is called completely excessive (with respect to $\left(P_{t}\right)$ ), if $\left(m P_{t}\right)_{t>0}$ is a completely monotone family of measures.

b) A $\sigma$-finite measure $m$ is called an eigenmeasure (with respect to $\left(P_{t}\right)$ ) for the eigenvalue $s \in\left[0, \infty\left[\right.\right.$ if $m P_{t}=e^{-s t} m$ for all $t>0$. Eigenmeasures for the eigenvalue 0 are called invariant measures. Obviously, eigenmeasures are completely excessive measures.

c) A $\sigma$-finite measure $m$ is called an ultra-potential measure (with respect to $\left(P_{t}\right)$ ) if for every $n$ in $\mathbb{N}$ we have $m=\mu_{n} U^{n}$ for some measure $\mu_{n}$ (here $U^{n}$ denotes the $n$-th iterate of the kernel $U$ ). Obviously, if $m$ is an eigenmeasure for a strictly positive eigenvalue, then $m$ is an ultrapotential measure.

(2.2) Theorem. Let $m$ be an excessive measure on $(E, \mathscr{E})$. Then the following properties are equivalent:

a) $m$ is completely excessive;

b) $m P_{t}=\int e^{-s t} \rho_{s} \sigma(d s)$ for all $t>0$, where the following property I holds:

I: $\sigma$ is a $\sigma$-finite measure on $[0, \infty[$, for $\sigma$-a.a. $s$ in $[0, \infty[$ the measure $\rho_{s}$ on $(E, \mathscr{E})$ is an eigenmeasure for the eigenvalue $s$, and $s \rightarrow \rho_{s}(f)$ is borel for every $f$ in $\mathscr{E}_{+}$;

c) $m=\int \rho_{s} \sigma(d s)$, where the above property I holds;

d) $m$ is the sum of an invariant measure and an ultra-potential measure.

Moreover, the measure $\int \rho_{s} \otimes \varepsilon_{s} \sigma(d s)$ on $E \times[0, \infty[$ in I is uniquely determined.

Proof. a) $\Rightarrow$ b). From (1.4) we find the measures $\sigma$ and $\left(\rho_{s}\right)_{s \geq 0}$ which give the representation of $\mathrm{b}$ ). To derive the property I we consider the two representations of $m P_{t} P_{h}=m P_{t+h}$ in b), i.e. 


$$
\int e^{-s t} \rho_{s} P_{h} \sigma(d s)=\int e^{-s(t+h)} \rho_{s} d \sigma(s) \quad \text { for all } t>0, h>0 .
$$

The uniqueness part in (1.4) implies for every $h>0$

$$
\rho_{s} P_{h}=e^{-s h} \rho_{s} \quad \text { for } \sigma \text {-a.a. } s \geq 0
$$

with an exceptional set depending on $h$. Consequently, $\{(s, h) \in[0, \infty[\times$ ]0, $\infty\left[: \rho_{s} P_{h} \neq e^{-s h} \rho_{s}\right\}$ is by Fubini's theorem negligeable for the product measure of $\sigma$ and Lebesgue measure on $] 0, \infty[$, hence we obtain for every $\lambda>0$

$$
\rho_{s} U_{2}=\frac{1}{\lambda+s} \rho_{s} \quad \text { for } \sigma \text {-a.a. } s \geq 0
$$

where the exceptional set of $s$ in $[0, \infty$ [ does not depend on $\lambda$. Replacing $\rho_{s}$ by the zero measure for these exceptional points gives the property I, since

$$
\rho U_{\lambda}=\frac{1}{\lambda+s} \rho \quad \text { for all } \lambda>0
$$

implies that $\rho$ is an excessive measure, hence $\left(\rho P_{t}(f)\right)_{t \geq 0}$ and $\left(e^{-s t} \rho(f)\right)_{t \geq 0}$ are for $\rho$-integrable functions $f$ right continuous on $\mathbb{R}_{+}$with the same Laplace transform, hence these two functions coincide.

b) $\Rightarrow c)$. By monotone convergence.

c) $\Rightarrow \mathrm{d})$. The measure $\sigma(\{0\}) \rho_{0}$ is invariant. By assumption we have for $\sigma$-a.a. $s \geq 0$ for all $t>0$

$$
\rho_{s} P_{t}=e^{-s t} \rho_{s},
$$

hence $s \rho_{s} U=\rho_{s}$, and by recurrence we conclude $s^{n} \rho_{s} U^{n}=\rho_{s}$, hence

$$
m-\sigma(\{0\}) \rho_{0}=\mu_{n} U^{n}
$$

for $\mu_{n}:=\int s^{n} \rho_{s} \sigma(d s)$ for all $n$ in $\mathbb{N}$.

d) $\Rightarrow$ a). This is obvious for invariant measures. Hence we have to show that ultra-potential measures are completely excessive. This follows easily from the equality

$$
\mu U P_{t}-\mu U P_{t+h}=\int \mu P_{u} d u \quad(t, h>0),
$$

which holds if $\mu U$ is a $\sigma$-finite measure. Finally, the stated unicity follows from the unicity in (1.4). 
(2.3) Remarks. a) M. Itô considers in [9] completely superharmonic measures. This class of measures, which (under strong regularity assumptions) is defined using the generator of the semigroup, contains the completely excessive measures as defined in (2.1). M. Itô gives an integral representation of completely superharmonic measures analogous to (2.2), where an additional term $\sum_{n \geq 1} \mu_{n} U^{n}$ with harmonic measures $\mu_{n}$ appears.

b) There is an obvious analogous notion of completely excessive functions. L. Beznea characterized in [4] completely excessive functions for a resolvent $\left(U_{k}\right)$, supposing hypothesis $L$ (i.e. there exists a reference measure for the resolvent) and an additional regularity assumption. In fact, it is well known, that under hypothesis $L$ excessive functions with respect to $\left(U_{2}\right)$ can be identified via the energy functional with excessive measures for some dual resolvent (cf. [5]). Consequently, under hypothesis $L$ our result (2.2) gives a characterization of completely excessive functions without L. Beznea's regularity assumption, if the following remark is carried out in detail.

c) Starting only with a resolvent $\left(U_{\lambda}\right)_{\lambda>0}$ of kernels (which does not come necessarily from a semigroup $\left(P_{t}\right)_{t>0}$ of kernels), one can characterize completely excessive measures $m$ by properties of $\left(m U_{\lambda}\right)_{\lambda>0}$ using Stieltjes transforms instead of Laplace transforms (cf. [3] and [4]).

d) A $\sigma$-finite measure $m$ on $(E, \mathscr{E})$ might be called completely supermedian with respect to $\left(P_{t}\right)$, if $\left(m P_{t}\right)_{t>0}$ is a completely monotone family of measures. Then a variant of (2.2) remains true for such measures, i.e. there is an additional term $\rho_{\infty}$ corresponding to an eigenvalue $s=\infty$, where $\rho_{\infty}$ is the purely supermedian measure given by the restriction of $m$ to the set $\{U 1=0\}$ (cf. Chap. XIII in [7]). This corresponds to Bernstein's theorem for completely monotone functions on $\mathbb{R}_{+}$instead of $] 0, \infty[$.

e) The above characterization tells nothing about the existence of non-trivial completely excessive measures. In fact, it is well known that under suitable ellipticity conditions on $\left(P_{t}\right)$ the set of eigenvalues is upper bounded; moreover, for large classes of elliptic differential operators on domains of $\mathbb{R}^{n}$ all ultra-potential measures are proportional (cf. [4] for details).

f) The existence of "many" eigenvalues for $\left(P_{t}\right)$ seems to be related with parabolicity. For example, if for a sequence $\left(s_{n}\right)$ tending to infinity there exist reference measures $\rho_{s_{n}}$ which are eigenmeasures for the eigenvalues $s_{n}$, then we have for all $\lambda>0$ 


$$
\rho_{s_{n}}=\lambda \int e^{-\lambda t} \rho_{s_{n}} d t=\lambda \int e^{-\lambda t} e^{s_{n} t} \rho_{s_{n}} P_{t} d t=\lambda \rho_{s_{n}} U_{\lambda-s_{n}} .
$$

If moreover for some strictly positive function $h$ in $\mathscr{E}_{+}$we have $\rho_{s_{n}}(h)<\infty$ for all $n$, then we conclude that $U_{1-s_{n}}(h)<\infty$ for all $n$ except on a fixed set of potential zero. This implies that $U$ is a parabolic potential kernel in the sense of [1]. It is an open question, if for parabolic potential kernels after a suitable time change such eigenvalues always exist.

Using a result of $M$. Itô in [9], we can also prove in our setting that the class of ultra-potential measures does not change if the original semigroup is changed by nice subordination in the sense of Bochner.

(2.4) Proposition. Let $\left(\eta_{t}\right)_{t \in 0}$ be a convolution semigroup of probability measures on $\left[0, \infty\left[\right.\right.$ such that $\kappa:=\int \eta_{t} d t$ has support $\left[0, \infty\left[\right.\right.$. Let $\left(P_{t}\right)_{t>0}$ be a measurable semigroup of kernel on a U-space $E$, and let $\left(Q_{t}\right)_{t>0}$ be the semigroup of kernels subordinated to $\left(P_{t}\right)$ in the sense of Bochner by $\left(\eta_{t}\right)$, i.e. $Q_{t}=\int P_{s} \eta_{t}(d s)$ for all $t>0$.

Then $\left(P_{t}\right)$ and $\left(Q_{t}\right)$ have the same ultra-potential measures.

Proof. By (2.2) it is enough to show that both semigroups have the same eigenmeasures for strictly positive eigenvalues.

a) Let $m$ be an excessive measure for $\left(P_{t}\right)$ such that $m P_{t}=e^{-\alpha \tau} m$ for all $t>0$ for some $\alpha>0$. Then

$$
m Q_{t}=\int m P_{s} \eta_{t}(d s)=m \cdot \int e^{-\alpha s} \eta_{t}(d s)=e^{-\beta t} m \quad \text { for all } t>0
$$

for some $\beta>0$ according to Theorem (9.18) in [3], hence $m$ is an eigenmeasure for $\left(Q_{t}\right)$ for the eigenvalue $\beta$.

b) For the converse let $m$ be an excessive measure for $\left(Q_{t}\right)$ such that $m Q_{t}=e^{-\beta t} m$ for all $t>0$ for some $\beta>0$. Since $P_{s}$ and $Q_{t}$ commute, we have $m P_{s} Q_{t}=e^{-\beta t} m P_{s}$ for all $s \geq 0, t>0$ (if we interpretate $m P_{0}$ as $m$ ). Hence for $f$ in $\mathscr{E}_{+}$, the function

$$
(*)
$$

$$
\varphi(s):=m P_{s} f \quad \text { for } s \geq 0
$$

satisfies

$$
\int \varphi(t+s) \eta_{u}(d t)=e^{-\beta u} \varphi(s) \quad \text { for all } u>0, s \geq 0
$$

From Remark 27 in [9] we conclude 


$$
\varphi(t)=c e^{-\alpha t} \quad \text { for } t \geq 0
$$

for suitable $c \geq 0$ and $\alpha>0$. From $(*)$ we conclude then that $c=\varphi(0)$ $=m(f)$, and $(* *)$ implies that $\alpha$ depends only on $\beta$ and on $\left(\eta_{u}\right), \alpha$ does not depend on $f$. All together we have $m P_{t}=e^{-a t} m$ for all $t \geq 0$, i.e. $m$ is an eigenmeasure for $\left(P_{t}\right)$.

\title{
REFERENCES
}

[1] Ben Saad, H., Janßen, K., A characterization of parabolic potential theory, Math. Ann., 272 (1985), 281-289.

[ 2 ] Berg, C., Christensen, J. P. R., Ressel, P., Harmonic analysis on semigroups, New York-Berlin-Heidelberg-Tokyo, Springer, 1984.

[ 3 ] Berg, C., Forst, G., Potential theory on locally compact abelian groups, BerlinHeidelberg-New York, Springer, 1975.

[4] Beznea, L., Ultrapotentials and positive eigenfunctions for an absolutely continuous resolvent of kernels, Nagoya Math. J., 112 (1988), 125-142.

[ 5 ] Boboc, N., Bucur, G., Cornea, A., Order and convexity in potential theory, Lecture notes in math., 853, Berlin-Heidelberg-New York, Springer, 1981.

[6 ] Dellacherie, C., Meyer, P. A., Probabilités et potentiel, Chap. I à IV. Paris, Hermann, 1975.

[ 7 ] — Probabilités et potentiel, Chap. XII à XVI. Paris, Hermann, 1987.

[ 8 ] Getoor, R. K., On the construction of kernels, Séminaire de Prob. de Strasbourg IX, 443-463, Lecture notes in math., 465. Berlin-Heidelberg-New York, Springer, 1975.

[ 9 ] Itô, M., Positive eigen elements for an infinitesimal generator of a diffusion semigroup and their integral representations, Potential theory Copenhagen 1979, 163184. Lecture notes in math., 787, Berlin-Heidelberg-New York, Springer, 1980.

[10] Itô, M., Suzuki, N., Completely superharmonic measures for the infinitesimal generator $\mathrm{A}$ of a diffusion semigroup and positive eigen elements of A, Nagoya Math. J., 83 (1981), 53-106.

[11] Janßen, K., Representation of excessive measures, Seminar on stochastic processes 1986, 85-105. Progress in probability and statistics, vol. 13. Boston-Basel-Stuttgart, Birkhäuser, 1987.

\author{
Hedi Ben Saad \\ Fac. des Sciences de Tunis \\ Dépt. de Mathématiques \\ 1060 Tunis-Belvedère \\ Tunisie \\ K. Janßen \\ Mathematisches \\ Institut der Universität Düsseldorf \\ Universitätsstr. 1 \\ D-4000 Düsseldorf
}

